\title{
Venezuelan hemorrhagic fever
}

INSERM

\section{Source}

INSERM. (1999). Orphanet: an online rare disease and orphan drug data base. Venezuelan hemorrhagic fever. ORPHA:319234

Venezuelan hemorrhagic fever (VHF), caused by the Guanarito virus, is a viral hemorrhagic disease characterized by fever, headache, arthralgia, sore throat, convulsions, and hemorrhagic manifestations. 